\title{
Active surfaces engineered by immobilizing protein-polymer nanoreactors for selectively detecting sugar alcohols
}

Xiaoyan Zhang' ${ }^{1}$, Mihai Lomora ${ }^{1}$, Tomaz Einfalt ${ }^{1}$, Wolfgang Meier ${ }^{1}$, Noreen Klein ${ }^{2}$, Dirk Schneider ${ }^{2}$, and Cornelia G. Palivan ${ }^{1 *}$

${ }^{1}$ Chemistry Department, University of Basel, Klingelbergstrasse 80, Basel, Switzerland

${ }^{2}$ Biochemistry, Institute of Pharmacy \& Biochemistry, University of Mainz, Germany

* Corresponding author. Tel.: +41 (0)61 26738 39; fax. +41 (0)61 2673855.

E-mail address: cornelia.palivan@unibas.ch (C.G. Palivan). 


\begin{abstract}
We introduce active surfaces generated by immobilizing protein-polymer nanoreactors on a solid support for sensitive sugar alcohols detection. First, such selective nanoreactors were engineered in solution by simultaneous encapsulation of specific enzymes in copolymer polymersomes, and insertion of membrane proteins for selective conduct of sugar alcohols. Despite the artificial surroundings, and the thickness of the copolymer membrane, functionality of reconstituted E. coli glycerol facilitator $(\mathrm{GlpF})$ was preserved, and allowed selective conduct of sugar alcohols to the inner cavity of the polymersome, where encapsulated ribitol dehydrogenase (RDH) enzymes served as biosensing entities. Ribitol, selected as a model sugar alcohol, was detected quantitatively by the RDH-nanoreactors with GlpF-mediated permeability in a concentration range of 1.5 - $9 \mathrm{mM}$. To obtain "active surfaces" for detecting sugar alcohols, the nanoreactors optimized in solution were then immobilized on a solid support: aldehyde groups exposed at the compartment external surface reacted via an aldehydeamino reaction with glass surfaces chemically modified with amino groups. The nanoreactors preserved their architecture and activity after immobilization on the glass surface, and represent active biosensing surfaces for selective detection of sugar alcohols, with high sensitivity.
\end{abstract}

Keywords: amphiphilic triblock copolymers; membrane proteins; membrane permeability; nanoreactors; active surfaces; selectivity. 


\section{Introduction}

The chemistry of saccharides and related compounds plays a significant role in the metabolic pathways of living organisms (e.g. to store energy, or to activate inflammatory mediators),[1] and their miss-regulation is involved in serious pathologic situations, such as diabetes, bloating and diarrhea.[2,3] In particular, sugar alcohols (e.g. D-sorbitol and D-mannitol) are essential metabolic intermediates involved in biological processes, such as maintaining renal, hepatic or neuro function, and have been used for the treatment of acute renal failure or in hepatic and neuro critical situations (e.g. mannitol has been reported as a neuroprotectant by scavenging free-radicals).[4-6] Therefore, the detection and concentration of sugar alcohols in aqueous solutions is necessary for a variety of applications, in biochemical, medical or even industrial domains. Most of the reported sensors for sugar alcohols are sweet taste sensors, based on interactions between hydrogen donor and receptor groups,[7] but are not directly specific for sugar alcohols. There are only very few examples of sensors for sugar alcohols based on biological selection or recognition.[8-10] To the best of our knowledge, only one type of synthetic sensor based on boronic acid molecular receptors that binds carbohydrates via covalent interactions in aqueous media has been reported as specific for sugar alcohols.[8-10]

An elegant way to detect specific molecules with high sensitivity is by designing nanoreactors, which encapsulate active molecules that sense their presence inside polymer supramolecular assemblies (polymersomes, micelles, dendrimers, and capsules) with sizes in the nanometer range.[11, 12] In particular, polymersome membranes formed via self-assembly of block copolymers have high mechanical robustness, and have greater temporal stability than the lipidbased membranes of liposomes.[11, 13-15] The propeties of polymersomes are controllable by the choice of monomer, and by adjusting the polymer molecular weight or their hydrophobic to hydrophilic ratio during synthesis.[11, 16, 17] The advantage of using polymer-based nanoreactors for detecting specific molecules is their ability to simultaneously protect the encapsulated active compound (enzyme, protein, mimic) from adverse environmental conditions, and allow it to act in situ for longer periods of time, compared with previously reported enzyme-loaded liposomes.[18] In addition, the intrinsic properties of polymer assemblies (stability, robustness, stimuli-responsivness), and of biological entities (specificity, rapidity and selectivity) favor their use in the development of detection systems with improved efficacy.[11-13] 
Nanoreactors for sensitive detection purposes have been developed by encapsulating single types of enzyme, such as acid phosphatase, inside polymersomes where, for example, they were able to produce $\mathrm{GdPO}_{3}$ as a contrast agent in MRI,[19] or an insoluble fluorescent product (ELF 97) for fluorescence detection in biological compartments with low pH.[14] A step further was achieved by co-encapsulating enzymes acting in tandem, for example for the simultaneous detection of superoxide radicals and their detoxification in situ inside the polymersome cavity.[12] Upon up-take by cells, the system acted as artificial organelles mimicking natural peroxisomes in detecting and combating superoxide radicals and $\mathrm{H}_{2} \mathrm{O}_{2}$, which are known to be associated with cellular oxidative stress.[17]

In order to provide rapid and specific responses that are crucial for biosensing applications, it is essential that membranes are permeable to substrates and products of reactions. Thus, inspired by natural cell membranes, our group pioneered an approach for controlling membrane permeability of polymersomes by the reconstitution of channel proteins (OmpF, LamB, AqpZ) in them, even though their thicknesses were significantly higher (3-6 times) than the size of the biomolecule.[15, 20-23] The high flexibility and the conformational freedom of the polymer chains allowed the polymersome membrane to adapt to the specific structural and dynamic properties of protein channels, supporting therefore a functional protein reconstitution.[24] However, insertion of channel proteins simply allowed passive and non-specific transport of molecules through the membranes, and this was limited only by the pore diameter. Permeable membranes have been also obtained by using copolymers that self-assemble in porous membranes (e.g. poly(styrene)-block-polyisocyanoalanine(2-thiophene-3-yl-ethyl) amide or boronic acid-based block copolymers),[25, 26] or by chemical modification of the polymer membrane with a hydroxyalkylphenone,[27] but none provided conditions for selective transport of molecules. Very recently membranes allowing selective passage of ions were introduced by inserting biopores, such as gramicidin, or ion carriers, such as ionomycin, and these were able to function as sensitive ion sensors.[23, 28] As the small diameter of biopores does not allow passage of molecules, membrane proteins serving in nature for passive or active transport of molecules are ideal candidates for selective permeabilization of membranes.

We describe here the design of polymer nanoreactors (PNRs) and their immobilization on a solid support to produce "active surfaces", which act as selective biosensors for sugar alcohols with both time and selectivity precision (Fig. 1). These nanoreactors are based on polymersomes in which specific membrane proteins are inserted in the membrane, and enzymes sensitive to sugar alcohols are encapsulated inside the cavity. The specific membrane proteins 
we selected are able to selectively allow diffusion of sugar alcohols into the inner cavity of the polymersomes, whilst the enzymatic reaction is used for sensitive and selective sugar alcohol biosensing. In order to generate PNRs, we chose poly(2-methyloxazoline)-blockpoly(dimethylsiloxane)-block-poly(2-methyloxazoline) (PMOXA- $b$-PDMS- $b$-PMOXA) copolymers because, depending on their hydrophilic-to-hydrophobic ratio, such amphiphilic copolymers self-assemble in dilute aqueous solutions, and generate polymersomes with sizes in the nanometer range.[21, 23, 29] The selected block copolymer features various properties that favor biomedical applications, such as good biocompatibility, low immunogenicity and toxicity, and a hydrophilic block with low ability for nonspecific protein binding.[30-32] In addition, polymersomes based on PMOXA- $b$-PDMS- $b$-PMOXA triblock copolymers both in solution,[17] and immobilised on solid support [33] have been already reported to be stable in complex biological environment (several days when immobilised on solid support). As a model channel protein for selective transport of sugar alcohols through the synthetic membrane, we selected the $E$. coli glycerol facilitator (GlpF), which conducts sugar alcohols with a high degree of stereo selectivity (Fig. 1A).[34-37] Inside the PNRs we encapsulated RDH, which is an enzyme that catalyzes a variety of sugar alcohol reactions, e.g. conversion of ribitol with oxidized nicotinamide adenine dinucleotide $\left(\mathrm{NAD}^{+}\right)$to D-ribulose, and reduced nicotinamide adenine dinucleotide (NADH).[38, 39] Therefore this "smart" combination of biomolecules serves to obtain a selective transport through the membrane of sugar alcohols, and their detection, as key point of the biosensor selectivity.

PNRs were then immobilized on planar solid supports to create "active surfaces" suitable for medical applications (Fig. 1B). Such "active surfaces" have several advantages, including: (i) high selectivity based on the membrane protein specificity for just certain molecules, (ii) long term stability of the encapsulated enzymes, which are protected from the hostile external environment, but are free to act in situ, (iii) controlled conditions for enzymatic reactions, which occur only in the confined cavity of nanoreactors, and (iv) precise spatial and temporal responses to the presence of the biological molecules to be detected.

\section{Materials and methods}

All chemicals and solvents were purchased from Sigma Aldrich (Sigma Chemical Co., USA), unless otherwise stated, at the highest purity grade and were used as received. 1-palmitoyl-2oleoyl-sn-glycero-3-phosphocholine (POPC) and 1-palmitoyl-2-oleoyl-sn-glycero-3-phospho$L$-serine (POPS) were purchased from Avanti Polar Lipids, Inc. (USA), Atto 488 maleimide 
from ATTO-TEC GmbH (Germany), and n-octyl- $\beta$-d-glucopyranoside ( $\beta$-OG) from Anatrace (USA). The glycerol facilitator $(\mathrm{GlpF})$ and outer membrane protein $\mathrm{F}(\mathrm{OmpF})$ were expressed in BL21 (DE3) Escherichia coli cells (Agilent Technologies, USA) as previously described (detailed information is provided in Supporting Information).[34, 40] His-tagged recombinant Enterobacter aerogenes ribitol 2-dehydrogenase (RDH) was acquired from Cusabio Biotech Co., Ltd (China). Bio-medium: Dubecco's Modified Eagle Medium with 10\% Fetal Bovine Serum and Penstrep.

\subsection{Preparation of GlpF-containing liposomes and PNRS}

GlpF-containing lipid vesicles were prepared by the film rehydration method as described previously.[23, 41] Briefly, a mixture solution of POPC and POPS with a weight ratio of 4:1 in $\mathrm{CHCl}_{3}$ was dried until a thin film was formed, and this was then rehydrated with PBS/protein solution to a final concentration of $1 \mathrm{mg} \mathrm{mL}^{-1}$.

Polymer nanorectors containing $\mathrm{OmpF} / \mathrm{GlpF}$ and $\mathrm{RDH}$ were prepared by a minor modification of the film rehydration method described previously.[40] $4 \mathrm{mg}$ of $\mathrm{PMOXA}_{6}-b-\mathrm{PDMS}_{42}-b-$ $\mathrm{PMOXA}_{6}$ copolymer were dissolved in $1 \mathrm{~mL}$ ethanol in round-bottom flasks, then placed in a rotary vacuum evaporator $\left(40^{\circ} \mathrm{C}, 1\right.$ hour, $170 \mathrm{rpm}$ for solvent removal) until a thin film was formed. The thin polymer film was rehydrated using $1 \mathrm{~mL}$ of $10 \mu \mathrm{g} \mathrm{mL}^{-1} \mathrm{RDH}$ solution in phosphate buffer solution ( $\mathrm{pH}$ 9), and $0.05 \mathrm{mg} \mathrm{ml}^{-1} \mathrm{GlpF}$ in $0.2 \% \mathrm{OG}$ and left over-night under magnetic stirring at $4{ }^{\circ} \mathrm{C}$. Polymersomes were extruded by passing them at least 11 times through $400 \mathrm{~nm}^{-1}$, followed by $200 \mathrm{~nm}$ - pore-size polycarbonate (PC) membranes (Merck, Germany) mounted in an Avanti mini-extruder (Avanti Polar Lipids, USA). To remove OG required to solubilize GlpF, and facilitate the protein insertion into the polymersome membrane, solutions were first dialyzed against PBS buffer at $4^{\circ} \mathrm{C}$ for 24 hours, changing the buffer every 8 hours, with molecular cut-off of 8-10 kDa MWCO dialysis membranes (Spectrum Laboratories, Inc. USA). Subsequently, solutions were dialyzed against PBS buffer at $4{ }^{\circ} \mathrm{C}$ for at least 24 hours, changing the buffer 4 times per day, using molecular cut-off of $300 \mathrm{kDa}$ MWCO dialysis membranes to remove the non-encapsulated RDH.

\subsection{Activity of PNR}

RDH activity was determined in bulk by spectrophotometric measurement of the change in absorbance of NADH at $340 \mathrm{~nm}$, using a standard assay mixture for substrate oxidation contained in a final volume of $1 \mathrm{~mL}$ at room temperature: $100 \mu \mathrm{mol}$ phosphate buffer solution 
at $\mathrm{pH} 9.0,1.8 \mu \mathrm{mol} \mathrm{NAD}{ }^{+}$and $0.02-0.2 \mathrm{U} \mathrm{RDH}$. The reaction was started by the addition of ribitol. One unit $(\mathrm{U})$ of enzyme activity is defined as the amount of enzyme required to oxidize $1 \mu \mathrm{mol} \mathrm{NAD}{ }^{+}$per min under standard assay conditions.

\subsection{Immobilization of PNRs}

PNRs able to be immobilized on a solid support were prepared as described above, but with a mixture of $5 \%$ aldehyde-terminated $\mathrm{PMOXA}_{7}-b-\mathrm{PDMS}_{44}-b-\mathrm{PMOXA}_{7}$ copolymer and $95 \%$ $\mathrm{PMOXA}_{6}-b$-PDMS $42-b-\mathrm{PMOXA}_{6}$ copolymer in PBS buffer containing a $0.3 \%(\mathrm{w} / \mathrm{v}) 2$-picoline borane complex at room temperature.

Immobilization of PNRs was performed by adapting to glass substrates (Thermos Scientific, Gerhard Menzel GmbH) a procedure described previously.[33] The amino functional groups exposed at the glass surface were obtained by immersing the glass surface in a solution of 3aminopropyltriethoxysilane (APTES) under water-free and oxygen-free conditions. Then, PNRs with exposed aldehyde groups were immobilized on functionalized glass surfaces by placing the glass substrates in a freshly-prepared PBS buffer solution containing PNRs and $0.3 \%$ sodium cyanoborohydride at room temperature. After $12 \mathrm{~h}$ of reaction, the solid substrates were extensively rinsed with bidistilled water and dried under a stream of nitrogen prior to scanning electron microscopy (SEM) or atomic force microscopy (AFM) measurements.

\subsection{Characterization techniques}

\subsubsection{Light scattering $(L S)$}

Dynamic (DLS) and static (SLS) light-scattering experiments were performed at $20 \pm 0.05^{\circ} \mathrm{C}$ on an ALV (Langen, Germany) goniometer equipped with an ALV He-Ne laser (JDS Uniphase, wavelength $\lambda=632.8 \mathrm{~nm}$ ). Polymersome solutions were placed in $10 \mathrm{~mm}$ cylindrical quartz cells, and measured at angles of $30-150^{\circ}$ using concentrations ranging from $0.4 \mathrm{mg} \mathrm{mL}^{-1}$ to $0.16 \mathrm{mg} \mathrm{mL}^{-1}$. The photon intensity autocorrelation function $\mathrm{g} 2(\mathrm{t})$ was determined with an ALV-5000E correlator. A non-linear decay-time analysis supported by regularized inverse Laplace transform of g2(t) (CONTIN algorithm) was used to analyze the DLS data. The angledependent apparent diffusion coefficient was extrapolated to zero momentum transfer (q2) using the ALV/Static and dynamic FIT and PLOT 4.31 software.[42]

\subsubsection{Transmission electron microscopy (TEM)}


To obtain TEM micrographs, $5 \mu \mathrm{L}$ of sample solutions were applied to freshly carbon-coated 200 mesh copper grids, followed by blotting the droplets on filter paper after $1 \mathrm{~min}$. Prior to measurements, a $2 \%$ uranyl acetate solution was used to negatively stain the samples. Grids were imaged using a Philips EM 400 (Philips Electronics, Netherlands), at $80 \mathrm{kV}$, and micrographs were processed by Morgagni 268D control and image acquisition software.

\subsubsection{Scanning electron microscopy (SEM)}

SEM was performed with a Hitachi S-4800 FEG (Hitachi, Ltd., Japan), operated at $5 \mathrm{kV}$ accelerating voltage. Before SEM measurements, the samples of PNRs or PNRs immobilized on glass surfaces were sputter-coated with platinum to minimize the overall change.

\subsubsection{Atomic force microscopy (AFM)}

AFM measurements were performed in a tapping mode under air using an Agilent 5100 AFM/SPM microscope (PicoLe System, Molecular Imaging). Images were recorded using silicon cantilevers (PPP-NCHR, Nanosensors) with a nominal spring constant of $42 \mathrm{~N} \mathrm{~m}^{-1}$, and collected with a resolution of $512 \times 512$ pixels at a scan rate of 0.5 line s${ }^{-1}$.

\subsection{5. $U V$-vis spectroscopy}

UV-Vis spectroscopy measurements were performed at $340 \mathrm{~nm}$ with a Specord 210 Plus spectrophotometer, Analytic Jena AG (10 mm quartz cuvettes), or a Spectramax M5e microplate reader, Molecular Devices (96-well plates).

\subsubsection{Stopped-flow spectroscopy}

Stopped-flow experiments were performed using a stopped-flow apparatus (Bio-Logic SAS, France) with a laser wavelength of $600 \mathrm{~nm}$. In a typical experiment, the vesicle suspension solution was placed in one reservoir/syringe of the instrument; each measurement was performed at $25^{\circ} \mathrm{C}$, using $140 \mu \mathrm{L}$ total volume of injected solution, $3.5 \mathrm{~mL} \mathrm{~s}^{-1}$ flow rate, and a 1:1 ratio of vesicle suspension to mixture solution. 5 individual runs were signal-averaged, and the resulting record saved for further analysis. The fluorescence detection of released 6carboxyfluorescein (self-quenched within the vesicles) with stopped-flow was measured using an excitation wavelength of $492 \mathrm{~nm}$, and a cut-off filter of $515 \mathrm{~nm}$.[43]

\subsubsection{Confocal laser scanning microscopy (CLSM)}


CLSM experiments were performed on a confocal laser-scanning microscope (Zeiss LSM 510META/Confocor2, Carl Zeiss, Jena, Germany) with a laser ( $\lambda=405 \mathrm{~nm}, 30 \mathrm{~mW}, 25 \%$ power output, $0.5 \%$ transmission) as excitation source. A main dichromatic beam splitter (HFT 488/543), a secondary dichroic beam splitter (NFT 515), and a band pass filter (BP 505-530) were used in all experiments. Each sample was scanned unidirectionally, and micrographs were recorded with a water immersion objective (C-Apochromat 40x/1.2W).

\subsubsection{Fluorescence correlation spectroscopy (FCS)}

All FCS measurements were performed using a Zeiss LSM 510-META/Confocor2 (Carl Zeiss, Jena, Germany) with an argon laser (488 $\mathrm{nm})$, and 40X water immersion C-Apochromat Objective lens. Measurements were performed at room temperature using a sample volume of $20 \mu \mathrm{L}$ on a covered eight-well Lab-TEk Nunc® Lab-Tek ${ }^{\circledR}$ II chambered cover glass (Nalage Nunc International). Measurements were recorded over 10s, and each measurement was repeated 15 times. The structural parameter and the diffusion time of the free dye (Atto-488) were determined independently. The autocorrelation function was calculated using a software correlator, and fitted with a one/two component(s) fit (LSM 510 META-ConfoCor 2 System). Detailed description of the FCS measurements and calculations are described in the SI.

\subsubsection{Ellipsometry}

Ellipsometry measurements were performed using an elliposometer (SE 850, Sentech instruments $\mathrm{GmbH}$, Germany) under air at room temperature. Prior to the measurement, the surface samples were thoroughly rinsed with water and dried under Ar flow. Each sample was measured under angle of $45^{\circ}, 55^{\circ}$ and $65^{\circ}$ using a wavelength range from $300 \mathrm{~nm}$ to $800 \mathrm{~nm}$. Data were fitted with a multilayer model for calculation of the film thickness.

\section{Results and discussion}

\subsection{Strategy for creating "active surfaces" based on PNRs}

The $\mathrm{PMOXA}_{6}-b$-PDMS ${ }_{42}-b$-PMOXA 6 copolymer we used here had already been shown to generate polymer vesicles with sizes in the nanometer range in which biopores could be successfully inserted,[23] or giant unilamellar vesicles (GUVs) for measuring the movement of natural channel proteins in artificial membranes.[29] PNRs with selective permeability were obtained by insertion of GlpF, and able to transport various sugar alcohols [34-37] into the membrane of the polymersomes. As GlpF had already been reconstituted in liposome 
membranes where it retained its natural function,[34-37] we first inserted the membrane protein into a mixture of POPC/POPS lipid membranes. The presence of GlpF and its purity were assessed by $10 \%$ SDS-PAGE (Supporting Information, Fig. S1), whilst its concentration was determined by measuring the absorbance intensity at $280 \mathrm{~nm}$ (molar extinction coefficient of $38305 \mathrm{M}^{-1} \mathrm{~cm}^{-1}$ ). In POPC-POPS liposomes, GlpF preserved its functionality upon insertion in the membrane (Supporting Information, Fig. S2).

For an enzyme able to sense the presence of sugar alcohols in situ, we selected ribitol dehydrogenase $(\mathrm{RDH})$ to encapsulate into the cavity of polymersomes.[38, 39] As we had already demonstrated the functional reconstitution of a channel porin OmpF in polymersome membranes [40], we initially produced PNRs by self-assembly of PMOXA6 6 -PDMS $42-b-$ $\mathrm{PMOXA}_{6}$ in the presence of $\mathrm{OmpF}$ and $\mathrm{RDH}$, in order to optimize conditions for encapsulating the enzyme. OmpF-PNRs were compared with empty polymersomes, and with unpermeabilised nanoreactors (PNRs without OmpF). Then, PNRs were formed by using GlpF instead of OmpF, using the optimized conditions for RDH encapsulation.

To obtain "active surfaces" for detecting sugar alcohols, PNRs were immobilized on glass solid supports, and their activities further investigated after immobilization; immobilization of nanoreactors is based on Schiff base formation between aldehyde groups exposed at the outer surface of nanoreactors and amino groups with which the support surface is functionalized.[33]

\subsection{Stability and functionality of GlpF in polymersome membranes}

According to the TEM micrographs, spherical nano-objects were detected after copolymer selfassembly, both without and with reconstituted GlpF, which indicates that GlpF did not affect the self-assembly process of $\mathrm{PMOXA}_{6}-b$-PDMS $42-b$-PMOXA 6 polymersomes (Fig. 2). They are in agreement with the cryoTEM results already reported for polymersomes obtained by selfassembly of this copolymer.[33] The visible shrinkage and wrinkle/sinking of the spherical objects indicated that they were hollow spherical nano objects - polymersomes - (without/with GlpF) affected by the drying procedure and high vacuum of the TEM set-up, as was previously reported.[33] The size distribution obtained by DLS indicates a population of spherical objects with average hydrodynamic radii of around $100 \mathrm{~nm}$, both without insertion of GlpF, and after insertion of GlpF (Table 1). These spherical objects have a hollow-sphere morphology because the values of the ratio $R_{g} / R_{h}$ of 1.05 (without GlpF), and 0.98 (with GlpF), respectively, are close to 1, which is specific for a hollow sphere architecture (Supporting Information Fig. S3).[23] This architecture is in good agreement with the aspect of the spherical objects in TEM. 
To assess conduct of sugar alcohols through GlpF-reconstituted liposome or polymersome membranes, we adapted an osmotic method, which gives information on the influx of molecules into vesicle cavities by stopped-flow spectrometry.[34, 36] GlpF has a high transport rate both for ribitol and glycerol,[36] but we selected ribitol as a model sugar alcohol to prove our biosensor concept because glycerol transport rates via GlpF are too fast for proper measurements.[36] Since the liposome and polymersome membranes are essentially impermeable to ribitol, the functionality of GlpF is determined by following the shrinking and re-swelling of the liposomes and polymersomes.

Upon mixture with a $400 \mathrm{mM}$ ribitol solution in phosphate-buffered saline (PBS, pH 8), GlpFcontaining polymersomes shrink immediately $(\leq 2$ s) due to an overall flow through the polymersomes under osmosis; this induces an increase in light scattering. The overall flow is based on a bidirectional process: water efflux out of the polymersomes, and ribitol influx inside them. Therefore, the slope of the curve corresponding to the water efflux from polymersomes without GlpF is steeper than that corresponding to water efflux, and ribitol influx from polymersomes with GlpF. A similar behavior has been obtained when liposomes without and with GlpF were measured (Supporting Information Fig. S2B).[34] When ribitol is diffusing into GlpF-containing polymersomes based on the functionality of GlpF, the polymersomes start to reswell because a water influx is following after 2-3 s (Fig. 3). Note that the polymer membranes we use are slightly permeable to water, because of the relatively short hydrophobic domain, as previously reported by stopped-flow spectroscopy.[44] The rate of ribitol influx is indicated by the speed of recovery of the initial volume of GlpF-containing polymersomes (faster ribitol influx results in quicker polymersome recovery). In contrast, when polymersome solutions without and with inserted GlpF were only mixed with the buffer, the size of the polymersomes remained constant (Supporting Information, Fig. S4 and Fig. 3). In addition, polymersomes without reconstituted GlpF when exposed to buffer solution of $400 \mathrm{mM}$ ribitol showed only an initial reduced water diffusion for less than $2 \mathrm{~s}$, associated to vesicle shrinkage, but no water re-swelling driven by the transport of the ribitol molecules through the GlpF pores, as in the case with polymersomes containing GlpF. As GlpF was initially stabilized by detergent (i.e. OG), polymersomes created in PBS buffer in the presence of detergent with a similar concentration to the GlpF solution,were also analyzed by stopped-flow spectroscopy. This showed that the presence of detergent had no effect on the permeability of the polymer membrane, (Supporting Information, Fig. S4). Whilst significantly more stable than liposomes (liposomes break in less than 5 seconds), the polymersomes are slowly starting to break due to 
the high osmotic pressure when they are exposed to $400 \mathrm{mM}$ ribitol after $1 \mathrm{~min}$ (Supporting Information, Fig. S5). High concentrations of ribitol are required for stop-flow experiments in order to get an appropriate and rapid response in time of the change in the polymersomes properties based on a significant difference in osmotic pressure. However, the ribitol concentration range intended to be detected by our PNRs is at least forty times lower than 400 $\mathrm{mM}$, and therefore the nanoreactors are stable for long periods of time in these conditions (see section 3.3). In addition, polymersomes mixted with ribitol solutions in a concentration range up to $10 \mathrm{mM}$ ribitol have a size change $<3 \%$ (Supporting Information, Fig. S6).

The comparison between the behavior of polymersomes without and with inserted GlpF with respect to ribitol influx is proof that the channel protein GlpF is functional inside the polymersome membrane despite its high thickness (10.3 nm [23]). Functional insertion of GlpF is supported by the high flexibility of copolymer chains inside the membrane, as already reported when gramicidin A was successfully inserted and remained functional in polymer membranes with thicknesses of 9.2 - $12.1 \mathrm{~nm}$, or when OmpF porin was successfully inserted into the same membrane of $10.3 \mathrm{~nm}$.[23, 40] The result is also in agreement with previously published data showing that small molecular weight polymer chains are more stretched inside the membrane than higher molecular weight chains that tend to adapt a random coil structure.[24] The stability and functionality of polymersomes with inserted GlpF has been tested after storage of 10 days: GlpF containing polymersomes preserved their function of ribitol diffusion (Supporting Information, Fig. S7).

The rate constant for reswelling polymersomes is dependent on the number of GlpF molecules reconstituted in the membrane, the osmotic gradient (sugar alcohol concentration), and the ability of GlpF to act as a selective channel for sugar alcohols. The fast reswelling speed of polymersomes containing GlpF $(<20 \mathrm{~s})$ indicates that the relatively high number of GlpF reconstituted in the synthetic membrane was sufficient to allow a fast ribitol flux.

\subsection{Characterization of PNRs}

A further step in obtaining active surfaces for sugar alcohol biosensing is the formation of nanoreactors by simultaneous insertion of $\mathrm{GlpF}$ in the polymersome membrane, and encapsulation of RDH in the inner cavity. TEM images demonstrate that the PNRs with inserted GlpF retain the morphology of polymersomes and GlpF containing polymersomes (Supporting Information, Fig. S8A), and even after two weeks of storage at room temperature or $37^{\circ} \mathrm{C}$, no significant morphological changes of the nanoreactor solutions appeared in TEM (Supporting 
Information, Fig. S8B) and SEM micrographs (Supporting Information, Fig. S9), suggesting that the PNRs with inserted GlpF are mechanically stable.

The in situ enzymatic activity of RDH was tested upon nanoreactor formation (Supporting Information Fig. S10). In order to exclude the possibility of enzyme adhesion on the surface, we used proteinase $\mathrm{K}$ - a member of the subtilisin-family, widely used in molecular biology to hydrolyze proteins. Due to the significant thickness of the polymer membrane $(10.3 \mathrm{~nm}$ for PMOXA $_{6}-b-\mathrm{PDMS}_{42}-b-\mathrm{PMOXA}_{6}$ membrane [23]), proteinase $\mathrm{K}$ was unable to hydrolyze/digest the reconstituted membrane proteins, which were protected by the hydrophilic domain of the membrane.[13] The sensitive reaction of RDH with ribitol, which produces $\mathrm{NADH}$, was monitored by the change in intensity of the UV/Vis absorbance at $340 \mathrm{~nm}$. As expected, the enzymatic reaction occurred only in the case of RDH encapsulated in GlpFcontaining polymersomes, because GlpF inserted in the membrane allowed ribitol to penetrate into the inner cavity (Fig. 4A). No enzymatic activity was detected in either RDH-loaded polymersomes without inserted GlpF, or the free enzyme in the presence of proteinase. Therefore, RDH was successfully encapsulated inside the nanoreactors, preserved its activity, and did not adhere to their external surface.

The encapsulation efficiency of Atto-488 labeled RDH was determined by FCS.[45] This was estimated as approx. 10\% (Supporting Information S11), in agreement with previous studies of single enzyme type encapsulation in polymersomes using the film rehydration method for nanoreactor preparation.[13, 46] The enzymatic activity of nanoreactors was further analyzed by qualitative comparison with that of the free enzyme at a similar concentration $(\sim 20 \mu \mathrm{g} \mathrm{mL}$ ${ }^{1}$ ). The encapsulated enzyme had slightly lower activity than the free enzyme (Fig. 5A and Supporting Information Fig. S9), which may be the consequence of slow diffusion of ribitol through the narrow GlpF channel. [36, 47] Indeed, when similar activity assays were performed using PNRs containing in their membrane the channel porin OmpF, which allows the passage of molecules smaller than $600 \mathrm{Da}[13,15,20]$, a higher reaction rate to ribitol was obtained (Supporting Information, Fig. S12). At the molecular level, the difference in the reaction rate between PNRs with inserted OmpF and PNRs with inserted GlpF is due to a different mode of the ribitol transport through the porin channel: in the case of GlpF, the polyalcohol flux is controlled by the stereo selective channel and conducted under a pull and push strength under membrane vibration, $[36,47]$ whereas the flux through OmpF is unspecific, and the molecular diffusion does not need energy because of the large pore of OmpF.[20, 48] 
Quantitative detection of ribitol with PNRs with inserted GlpF was possible for a concentration range of $1.5-9 \mathrm{mM}$ (Fig. 4B), where the change in absorbance intensity was proportional to the amount of added ribitol (reaction time $15 \mathrm{~min}$ ). All endpoints were in agreement with the theoretical values calculated using the extinction coefficient of NADH at $340 \mathrm{~nm}$, an important characteristic for an analytical use of our biosensor. The $K_{m}$ value $(7.9 \mathrm{mM})$ of the reaction of the encapsulated RDH with ribitol is in agreement with the values previously obtained for free $\mathrm{RDH},[49]$ which indicates that the encapsulated enzyme preserved its efficacy. Since the concentrations of sugar alcohols in biology or foods are in the milli-molar range, this biosensor has appropriate functionality for the development of medical applications.[3]

In addition, PNRs were stable for 5 days under the detection conditions, and in the presence of ribitol, up to a concentration of $10 \mathrm{mM}$, as indicated by TEM micrographs (Supporting Information, Fig. S13). As the normal time for biosensing purpose is orders of magnitude lower (few minutes), our PNRs with inserted GlpF are very robust and stable, supporting further development for applications. In addition, they are more advantageous for engineering biosensing systems in terms of membrane mechanical stability than liposomes (Fig. S14).

\section{4. "Active surface" characterization}

To obtain "active surfaces" for a rapid detection of sugar alcohols, PNRs were immobilized on a solid support. For this purpose polymersomes were self-assembled from a mixture of nonfunctionalized copolymers $\left(\mathrm{PMOXA}_{6}-b-\mathrm{PDMS}_{42}-b-\mathrm{PMOXA}_{6}\right)$ and $5 \%$ functionalized copolymers $\left(\mathrm{PMOXA}_{7}-b-\mathrm{PDMS}_{44}-b-\mathrm{PMOXA}_{7}\right)$ with aldehyde end groups to provide the possibility of immobilizing polymersomes onto glass surfaces chemically modified with amino groups via an aldehyde-amino reaction. Both the morphology of GlpF-containing polymersomes self-assembled from a mixture of non-functionalized copolymers and 5\% aldehyde-functionalized copolymers (used for the development of nanoreactors), and the GlpF functionality inside the synthetic membrane were similar to those of GlpF-containing polymersomes based on nonfunctionalized copolymers (Fig. 2, Fig. 3 and Supporting Information, Fig. S15). Therefore the presence of the aldehyde groups did not affect the protein functionality because both their low amount used for development of nanoreactors, and the low accessibility of the primary amines of GlpF (mainly found at the N-terminus and in the sidechain of lysines, Supporting Information, Fig. S16). After immobilizing the nanoreactors on the glass surface, the surface was rinsed several times with bidistilled water in order to remove any unimmobilized polymersomes. ATR-IR bands characteristic of the polymer backbones were 
observed for APTES modified glass substrates incubated with nanoreactors with aldehyde groups exposed at their surface, and indicated successful, covalent immobilization of the nanoreactors (Supporting Information Fig. S17). Ellipsometry measurements on various steps of surface functionalization indicated a successful APTES modification and then nanoreactors immobilization (Supporting Information, Tab. S1).

Both SEM and AFM images recorded randomly throughout the surface containing immobilized PNRs with inserted GlpF indicate the presence of spherical objects (Fig. 5). SEM micrographs (Fig. 5A) reveal that the solid surface was in homogeneously covered by spherical objects with diameters around $100-150 \mathrm{~nm}$, in agreement with the AFM scan under air (Fig. 5B). Rinsing the surface with bidistilled water did not remove the attached nanoreactors, which were covalently bound via amine bonds. In addition, the height of the collapsed polymersomes based on the magnified height images and the corresponding cross-section profiles (Fig. 5C), was determined to be $\sim 7 \mathrm{~nm}$, which is close to the thickness of two overlapping polymer layers in the dry state. The difference in the height curve of two selected nanoreactors (Fig. 5C) is due to the inherent size distribution of polymersomes. Note that the hydrophilic blocks of the copolymer retract and entangle, resulting in a lower value of the membrane thickness as compared with the reported value from cryoTEM in which the polymersomes were in an aqueous environment prior to their freezing.[29] The vesicular structures on the surface were not disrupted over an extended period of time: even after 10 days of storage in PBS buffer, immobilized nanoreactors were still present on the surface (Supporting Information, Fig. S18). Their covalent attachment provided high stability against Brownian motion and convection. These active surfaces preserved their morphology without breaking the immobilized nanoreactors when immersed in a bio-medium for more than 5 days (Supporting Information, Fig. S19).

As Ribitol Dehydrogenase (aprox. $118 \mathrm{kDa}$ ) has in solution a hydrodynamic radius of about 4.0 $\mathrm{nm}$ [50], it is expected to be completely embedded into the synthetic matrix of the collapsed polymersomes, and therefore no significant difference in the thickness of the collapsed PNRs with inserted GlpF compared with empty polymersomes has been observed when measured by AFM (in the limit of AFM resolution). Negligible difference in the roughness of the active surfaces $\left(R_{s q}\right.$ of $\left.4.21 \mathrm{~nm}\right)$ compared with surfaces with immobilized polymersomes $\left(R_{s q}\right.$ of 4.47 $\mathrm{nm}$ ) has been obtained (Supporting Information, Fig. S20).

\section{5. "Active surface" functionality}


As the enzyme reaction produces NADH, which is fluorescent (with an emission wavelength at $445 \mathrm{~nm}$ ), it was used as a probe to evaluate the activity after immobilization of PNRs with inserted GlpF on a solid support by confocal laser scanning microscopy (CLSM). CLSM micrographs of the active surface based on immobilized nanoreactors with GlpF showed a bright fluorescence of the surface (Fig. 6A) compared with the surface with immobilized nanoreactors without GlpF (Fig. 6B), and with the surface with nanoreactors with GlpF based on nonfunctional copolymers, which were easily rinsed away by water (Fig. 6C). Fouling properties of these polymersomes can be neglected both because of the small timescale of the detection, and the presence of PMOXA block exposed at the exterior of our PNRs. Overall nonfouling property of the surface was expected because of the well-known nonfouling propriety of PMOXA domain.[51] In addition, the cross scratch part of the surface, where no nanoreactors were present after rinsing with water, showed no fluorescence (Fig. 6A). Note, that although the excitation wavelength peak for NADH is $350-360 \mathrm{~nm}$, a low fluorescence emission is still able to be observed with the excitation wavelength at $405 \mathrm{~nm}$ (Supporting Information Fig. S21). Therefore, PNRs preserved their enzymatic activity after immobilization on the glass surface, and thus could function as active biosensing surfaces for sugar alcohols.

In addition, to address the performance of the active surface in terms of sensitivity and reproducibility, we immobilized nanoreactors with inserted GlpF on the interior wall of glass cuvettes, and measured the intensity of the fluorescence signal of NADH produced inside the PNRs with GlpF when the surface was exposed to increasing ribitol concentrations $(0-500 \mathrm{nM})$ (Supporting Information Fig. S22). The lowest concentration of ribitol detected by the active surface was $200 \mathrm{nM}$. In addition, UV/Vis spectroscopy was used to evaluate the active surface performance: a linear biosensing reply was obtained for milli-molar range of ribitol (Fig 7), in agreement with the performance of our PNRs in solution.

One of the main advantages of this surface-based bioanalytical sensor technology is the possibility of rapidly screening multiple recognition events either sequentially or simultaneously. Due to the flexible size of the glass cover slides that can be functionalised with immobilised PNRs with inserted GlpF, our active surfaces provide an ideal platform to be further developed in combination with "lab on a chip" devices [52] for translational applications.

\section{Conclusions}


We have developed "active surfaces" for selective biosensing of sugar alcohols with time and space precision based on immobilization of nanoreactors with specific membrane proteins inserted in their membranes, and sugar alcohol sensitive enzymes encapsulated in their cavities. Interestingly, the artificial surrounding within such a copolymer membrane, and the high membrane thickness did not affect the functionality of the reconstituted GlpF, which allowed selective flux of sugar alcohols into the inner cavity of the PNRs, where the encapsulated enzymes serve as biosensing entities. An advantage of encapsulating the enzymes inside polymersomes is their protection from a potentially harmful environment, whilst preserving their catalytic activity: this smart approach prolongs the life-time of the enzymatic biosensor. Such protein-polymer nanoreactors with selective permeability offer the opportunity of monitoring the concentration of biologically relevant sugar alcohols because GlpF is able to conduct other sugar alcohols as well, and therefore support biosensing approaches in a variety of domains, such as medical, biochemical or industrial. In addition, their immobilization on solid supports provides efficient "active surfaces" due to the rapid change in their fluorescence intensity in the presence of sugar alcohols. The concept of polymer compartments with selective permeability by inserting specific membrane proteins can be easily extended to the biosensing of other molecules by a simple change of membrane protein and encapsulated enzyme.

\section{Acknowledgements}

We gratefully acknowledge the financial support provided by the Swiss National Science Foundation, the Swiss Nanoscience Institute, and the National Centre of Competence in Research "Molecular Systems Engineering”. D. Schneider and N. Klein were supported by grants from the "Stiftung Rheinland-Pfalz für Innovation", the Research Center "Complex Materials" (COMATT) and the Funds of the German Chemical Industry. We thank Samuel Lörcher and Dr. Adrian Dinu (University of Basel) for polymer synthesis, Gabriele Persy (University of Basel) for TEM measurements, Martina Garni (University of Basel) for CLSM experiments, and Dr. Gesine Gunkel-Grabole (University of Basel) for AFM experiments. We acknowledge Dr. Elisa Nogueira for the work related to the molecular biology, and Dr. Marc Creus (University of Basel) for very fruitful discussions regarding the plasmid. Authors also thank Dr. B. A. Goodman for reading the manuscript. 


\section{References}

[1] Nelson ED, Ramberg JE, Best T, Sinnott RA. Neurologic effects of exogenous saccharides: A review of controlled human, animal, and in vitro studies. Nutr Neurosci. 2012;15:149-62.

[2] Parmpi P, Kofinas P. Biomimetic glucose recognition using molecularly imprinted polymer hydrogels. Biomaterials. 2004;25:1969-73.

[3] Ruskone-Fourmestraux A, Attar A, Chassard D, Coffin B, Bornet F, Bouhnik Y. A digestive tolerance study of maltitol after occasional and regular consumption in healthy humans. Eur J Clin Nutr. 2003;57:26-30.

[4] Tarczynski MC, Jensen RG, Bohnert HJ. Stress Protection of Transgenic Tobacco by Production of the Osmolyte Mannitol. Science. 1993;259:508-10.

[5] Bereczki D, Mihalka L, Szatmari S, Fekete K, Di Cesar D, Fulesdi B, et al. Mannitol use in acute stroke - Case fatality at 30 days and 1 year. Stroke. 2003;34:1730-5.

[6] Huang ZC, Dong WW, Yan Y, Xiao QF, Man YA. Effects of intravenous mannitol on EEG recordings in stroke patients. Clin Neurophysiol. 2002;113:446-53.

[7] Habara M, Ikezaki H, Toko K. Study of sweet taste evaluation using taste sensor with lipid/polymer membranes. Biosens Bioelectron. 2004;19:1559-63.

[8] James TD, Shinmori H, Shinkai S. Novel fluorescence sensor for 'small' saccharides. Chem Commun. 1997:71-2.

[9] Zhao JZ, James TD. Chemoselective and enantioselective fluorescent recognition of sugar alcohols by a bisboronic acid receptor. J Mater Chem. 2005;15:2896-901.

[10] Liang XF, James TD, Zhao JZ. 6,6 '-Bis-substituted BINOL boronic acids as enantio selective and chemoselective fluorescent chemosensors for D-sorbitol. Tetrahedron. 2008;64:1309-15.

[11] Palivan CG, Fischer-Onaca O, Delcea M, Itel F, Meier W. Protein-polymer nanoreactors for medical applications. Chem Soc Rev. 2012;41:2800-23.

[12] Tanner P, Egli S, Balasubramanian V, Onaca O, Palivan CG, Meier W. Can polymeric vesicles that confine enzymatic reactions act as simplified organelles? Febs Lett. 2011;585:1699-706.

[13] Langowska K, Palivan CG, Meier W. Polymer nanoreactors shown to produce and release antibiotics locally. Chem Commun. 2013;49:128-30.

[14] Broz P, Driamov S, Ziegler J, Ben-Haim N, Marsch S, Meier W, et al. Toward intelligent nanosize bioreactors: A $\mathrm{pH}$-switchable, channel-equipped, functional polymer nanocontainer. Nano Lett. 2006;6:2349-53.

[15] Nardin C, Thoeni S, Widmer J, Winterhalter M, Meier W. Nanoreactors based on (polymerized) ABA-triblock copolymer vesicles. Chem Commun. 2000:1433-4.

[16] Tanner P, Baumann P, Enea R, Onaca O, Palivan C, Meier W. Polymeric Vesicles: From Drug Carriers to Nanoreactors and Artificial Organelles. Accounts Chem Res. 2011;44:103949.

[17] Tanner P, Balasubramanian V, Palivan CG. Aiding Nature's Organelles: Artificial Peroxisomes Play Their Role. Nano Lett. 2013;13:2875-83.

[18] Walde P, Ichikawa S. Enzymes inside lipid vesicles: Preparation, reactivity and applications. Biomol Eng. 2001;18:143-77. 
[19] Broz P, Ben-Haim N, Santini F, Marsch S, Scheffler K, Meier W, et al. Inhibition of macrophage phagocytotic activity by a receptor-targeted polymer vesicle-based drug delivery formulation of pravastatin. Nanomedicine-Uk. 2009;2:43.

[20] Meier W, Nardin C, Winterhalter M. Reconstitution of channel proteins in (polymerized) ABA triblock copolymer membranes. Angew Chem Int Edit. 2000;39:4599-4602.

[21] Nardin C, Hirt T, Leukel J, Meier W. Polymerized ABA triblock copolymer vesicles. Langmuir. 2000;16:1035-41.

[22] Kumar M, Grzelakowski M, Zilles J, Clark M, Meier W. Highly permeable polymeric membranes based on the incorporation of the functional water channel protein Aquaporin Z. P Natl Acad Sci USA. 2007;104:20719-24.

[23] Lomora M, Garni M, Itel F, Tanner P, Spulber M, Palivan CG. Polymersomes with engineered ion selective permeability as stimuli-responsive nanocompartments with preserved architecture. Biomaterials. 2015;53:406-14.

[24] Itel F, Chami M, Najer A, Lorcher S, Wu DL, Dinu IA, et al. Molecular Organization and Dynamics in Polymersome Membranes: A Lateral Diffusion Study. Macromolecules. 2014;47:7588-96.

[25] Kuiper SM, Nallani M, Vriezema DM, Cornelissen JJLM, van Hest JCM, Nolte RJM, et al. Enzymes containing porous polymersomes as nano reaction vessels for cascade reactions. Org Biomol Chem. 2008;6:4315-8.

[26] Roy D, Sumerlin BS. Glucose-Sensitivity of Boronic Acid Block Copolymers at Physiological pH. Acs Macro Lett. 2012;1:529-32.

[27] Spulber M, Najer A, Winkelbach K, Glaied O, Waser M, Pieles U, et al. Photoreaction of a Hydroxyalkyphenone with the Membrane of Polymersomes: A Versatile Method To Generate Semipermeable Nanoreactors. J Am Chem Soc. 2013;135:9204-12.

[28] Lomora M, Itel F, Dinu IA, Palivan CG. Selective ion-permeable membranes by insertion of biopores into polymersomes. Phys Chem Chem Phys. 2015;17:15538-46.

[29] Itel F, Najer A, Palivan CG, Meier W. Dynamics of Membrane Proteins within Synthetic Polymer Membranes with Large Hydrophobic Mismatch. Nano Lett. 2015;15:3871-8.

[30] Broz P, Benito SM, Saw C, Burger P, Heider H, Pfisterer M, et al. Cell targeting by a generic receptor-targeted polymer nanocontainer platform. J Control Release. 2005;102:47588.

[31] De Vocht C, Ranquin A, Willaert R, Van Ginderachter JA, Vanhaecke T, Rogiers V, et al. Assessment of stability, toxicity and immunogenicity of new polymeric nanoreactors for use in enzyme replacement therapy of MNGIE. J Control Release. 2009;137:246-54.

[32] Pidhatika B, Rodenstein M, Chen Y, Rakhmatullina E, Muhlebach A, Acikgoz C, et al. Comparative Stability Studies of Poly(2-methyl-2-oxazoline) and Poly(ethylene glycol) Brush Coatings. Biointerphases. 2012;7.

[33] Langowska K, Kowal J, Palivan CG, Meier W. A general strategy for creating selfdefending surfaces for controlled drug production for long periods of time. J Mater Chem B. 2014;2:4684-93.

[34] Cymer F, Schneider D. A Single Glutamate Residue Controls the Oligomerization, Function, and Stability of the Aquaglyceroporin GlpF. Biochemistry-Us. 2010;49:279-86. 
[35] Veerappan A, Cymer F, Klein N, Schneider D. The Tetrameric alpha-Helical Membrane Protein GlpF Unfolds via a Dimeric Folding Intermediate. Biochemistry-Us. 2011;50:1022330.

[36] Fu DX, Libson A, Miercke LJW, Weitzman C, Nollert P, Krucinski J, et al. Structure of a glycerol-conducting channel and the basis for its selectivity. Science. 2000;290:481-6. [37] Borgnia MJ, Agre P. Reconstitution and functional comparison of purified GlpF and AqpZ, the glycerol and water channels from Escherichia coli. P Natl Acad Sci USA. 2001;98:2888-93.

[38] Poonperm W, Takata G, Izumori K. Polyol conversion specificity of Bacillus pallidus. Biosci Biotech Bioch. 2008;72:231-5.

[39] Moon HJ, Tiwari MK, Singh R, Kang YC, Lee JK. Molecular Determinants of the Cofactor Specificity of Ribitol Dehydrogenase, a Short-Chain Dehydrogenase/Reductase. Appl Environ Microb. 2012;78:3079-86.

[40] Einfalt T, Goers R, Dinu IA, Najer A, Spulber M, Onaca O, et al. Stimuli-Triggered Activity of Nanoreactors by Biomimetic Engineering Polymer Membranes. Nano Lett. 2015;15:7595-603.

[41] Spulber M, Baumann P, Saxer SS, Pieles U, Meier W, Bruns N. Poly(Nvinylpyrrolidone)-Poly(dimethylsiloxane)-Based Polymersome Nanoreactors for LaccaseCatalyzed Biotransformations. Biomacromolecules. 2014;15:1469-75.

[42] Baumann P, Balasubramanian V, Onaca-Fischer O, Sienkiewicz A, Palivan CG. Lightresponsive polymer nanoreactors: a source of reactive oxygen species on demand. Nanoscale. 2013;5:217-24.

[43] Raychaudhuri P, Li QH, Mason A, Mikhailova E, Heron AJ, Bayley H. Fluorinated Amphiphiles Control the Insertion of alpha-Hemolysin Pores into Lipid Bilayers.

Biochemistry-Us. 2011;50:1599-606.

[44] Najer A, Wu DL, Bieri A, Brand F, Palivan CG, Beck HP, et al. Nanomimics of Host Cell Membranes Block Invasion and Expose Invasive Malaria Parasites. Acs Nano. 2014;8:12560-71.

[45] Tanner P, Onaca O, Balasubramanian V, Meier W, Palivan CG. Enzymatic Cascade Reactions inside Polymeric Nanocontainers: A Means to Combat Oxidative Stress. Chem-Eur J. 2011;17:4552-60.

[46] Ranquin A, Versees W, Meier W, Steyaert J, Van Gelder P. Therapeutic nanoreactors: Combining chemistry and biology in a novel triblock copolymer drug delivery system. Nano Lett. 2005;5:2220-4.

[47] Grayson P, Tajkhorshid E, Schulten K. Mechanisms of selectivity in channels and enzymes studied with interactive molecular dynamics. Biophys J. 2003;85:36-48.

[48] Siti W, de Hoog HPM, Fischer O, Shan WY, Tomczak N, Nallani M, et al. An intercompartmental enzymatic cascade reaction in channel-equipped polymersome-inpolymersome architectures. J Mater Chem B. 2014;2:2733-7.

[49] Moon HJ, Tiwari M, Jeya M, Lee JK. Cloning and characterization of a ribitol dehydrogenase from Zymomonas mobilis. Appl Microbiol Biot. 2010;87:205-14. [50] Erickson HP. Size and Shape of Protein Molecules at the Nanometer Level Determined by Sedimentation, Gel Filtration, and Electron Microscopy. Biol Proced Online. 2009;11:3251. 
[51] Konradi R, Pidhatika B, Muhlebach A, Textort M. Poly-2-methyl-2-oxazoline: A peptide-like polymer for protein-repellent surfaces. Langmuir. 2008;24:613-6.

[52] Pennathur S, Fygenson DK. Improving fluorescence detection in lab on chip devices. Lab Chip. 2008;8:649-52. 


\section{Figure and Table Captions}

\section{Figure 1}

Figure 1 A) Molecular representation of the selective membrane protein GlpF (green) and ribitol as a model sugar alcohol (red): side view (upper part of the image), front view (lower part). The image depicts a static representation of a ribitol molecule that is able to translocate through a GlpF channel protein. B) Schematic representation of an "active surface" serving as a sugar alcohol biosensor based on immobilized protein-polymersome nanoreactors with reconstituted membrane protein $(\mathrm{GlpF})$ for selective transport of sugar alcohols, and encapsulated enzymes $(\mathrm{RDH})$ for sensitive detectionof sugar alcohols.

\section{Figure 2}

Figure 2 A) TEM micrographs of polymersomes without GlpF and B) with reconstituted GlpF. Insert in figure A, shows a broken polymersome, as a proof of the hollow inner cavity of the vesicular structures.

\section{Figure 3}

Figure 3 PNRs size variation due to changes in the osmotic pressure of the environment of polymersomes (shown through the schematic representation) by stopped flow spectroscopy: polymersomes mixed with a 400 mMribitol solution in PBS (black $\square$ ), GlpF-polymersome solution mixed with PBS buffer (blue $\Delta$ ), and GlpF-polymersomes mixed with a $400 \mathrm{mM}$ ribitol solution in PBS (red ○). GlpF-containing polymersomes shrink initially under PBS buffer containing ribitol, due to diffusion of water molecules through the polymersome membrane under osmotic pressure. Re-swelling depends on ribitol flux into the polymersome cavities.

\section{Figure 4}

Figure 4 A) Absorbance intensity measured at $340 \mathrm{~nm}$ when ribitol was mixed with: nanoreactors with inserted GlpF (red open $\boldsymbol{\nabla}$ ), nanoreactors without inserted GlpF (black $\square$ ), and free $\mathrm{RDH}$ in the presence of proteinase $\mathrm{K}$ (grey $\Delta$ ). B) Conversion of ribitol by nanoreactors with inserted GlpF in solution with ribitol at different concentrations: $1.5 \mathrm{mM}$ (black $\square$ ), $3 \mathrm{mM}$ (blue $\Delta$ ), $6 \mathrm{mM}$ (red $\circ$ ) and $9 \mathrm{mM}$ (pink open $\boldsymbol{\nabla}$ ). Substrate conversions were performed in the standard assay system at $\mathrm{pH} \mathrm{9,} \mathrm{and} \mathrm{the} \mathrm{change} \mathrm{in} \mathrm{the} \mathrm{produced} \mathrm{NADH} \mathrm{was} \mathrm{recorded} \mathrm{as}$ absorbance intensity at $340 \mathrm{~nm}$. Inset represents the reciprocal of the initial reaction speed (1/V) as function of the reciprocal of molar concentration of ribitol.

\section{Figure 5}


Figure 5 A) SEM images of nanoreactors with inserted GlpF covalently immobilized on an amino functionalized glass surface; B) Overview AFM image of the immobilized nanoreactors with inserted GlpF on an amino functionalized glass surface, and C) height curve of two selected nanoreactors.

\section{Figure 6}

Figure 6 CLSM micrographs of A) the active surface with immobilized PNRs with inserted GlpF, B) the surface with PNRs without inserted GlpF, and C) PNRs based on nonfunctional copolymers, which cannot be immobilized on glass and are easily rinsed away by water.

\section{Figure 7}

Figure 7 Absorbance intensity of NADH produced by the enzymatic reaction inside PNRs with GlpF immobilized on the interior wall of a glass cuvette, in the presence of $0.0-3.0 \mathrm{mM}$ ribitol $( \pm \mathrm{SD}, \mathrm{n}=3)$.

Table 1 Table 1 Static and dynamic light scattering data for polymersomes without and with GlpF. 
Table 1 Static and dynamic light scattering data for polymersomes without and with GlpF. (1.5 columns)

\begin{tabular}{lccccc}
\hline & $R_{g}$ & $R_{h}$ & $M_{w} \mathrm{~g} \mathrm{~mol}^{-1}$ & $A_{2} \mathrm{~mol} \mathrm{dm}^{3} \mathrm{~g}^{-2}$ & $R_{g} / R_{h}$ \\
\hline Polymersomes & $146 \pm 8$ & $138 \pm 3$ & $8.45 \times 10^{8}$ & $-1.804 \times 10^{-10}$ & 1.05 \\
Polymersomes with GlpF & $149 \pm 3$ & $152 \pm 2$ & $8.55 \times 10^{8}$ & $-1.114 \times 10^{-10}$ & 0.98
\end{tabular}


A
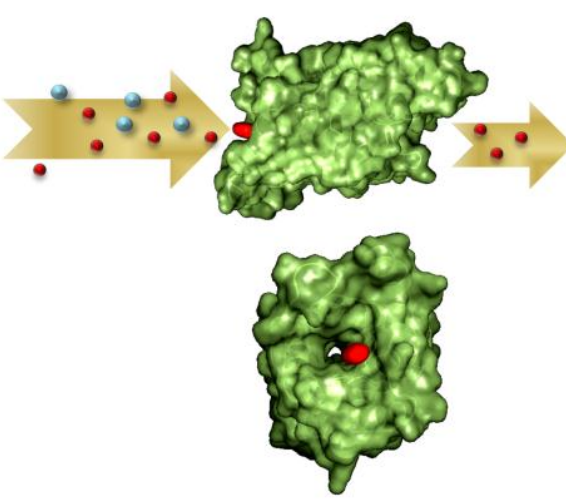

B

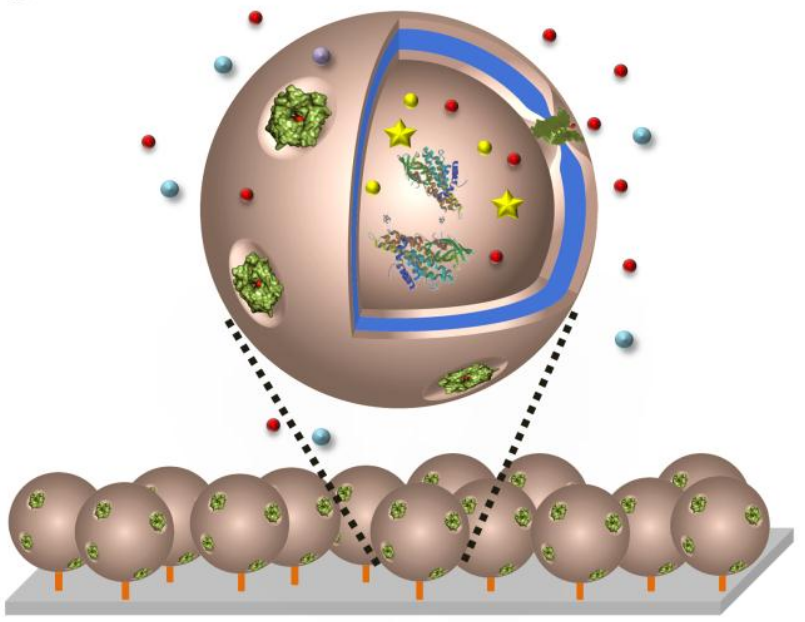



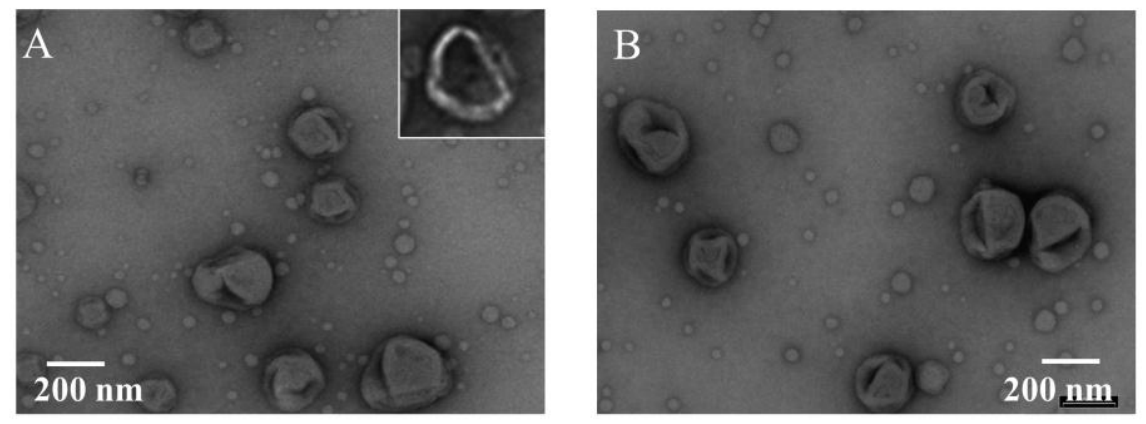


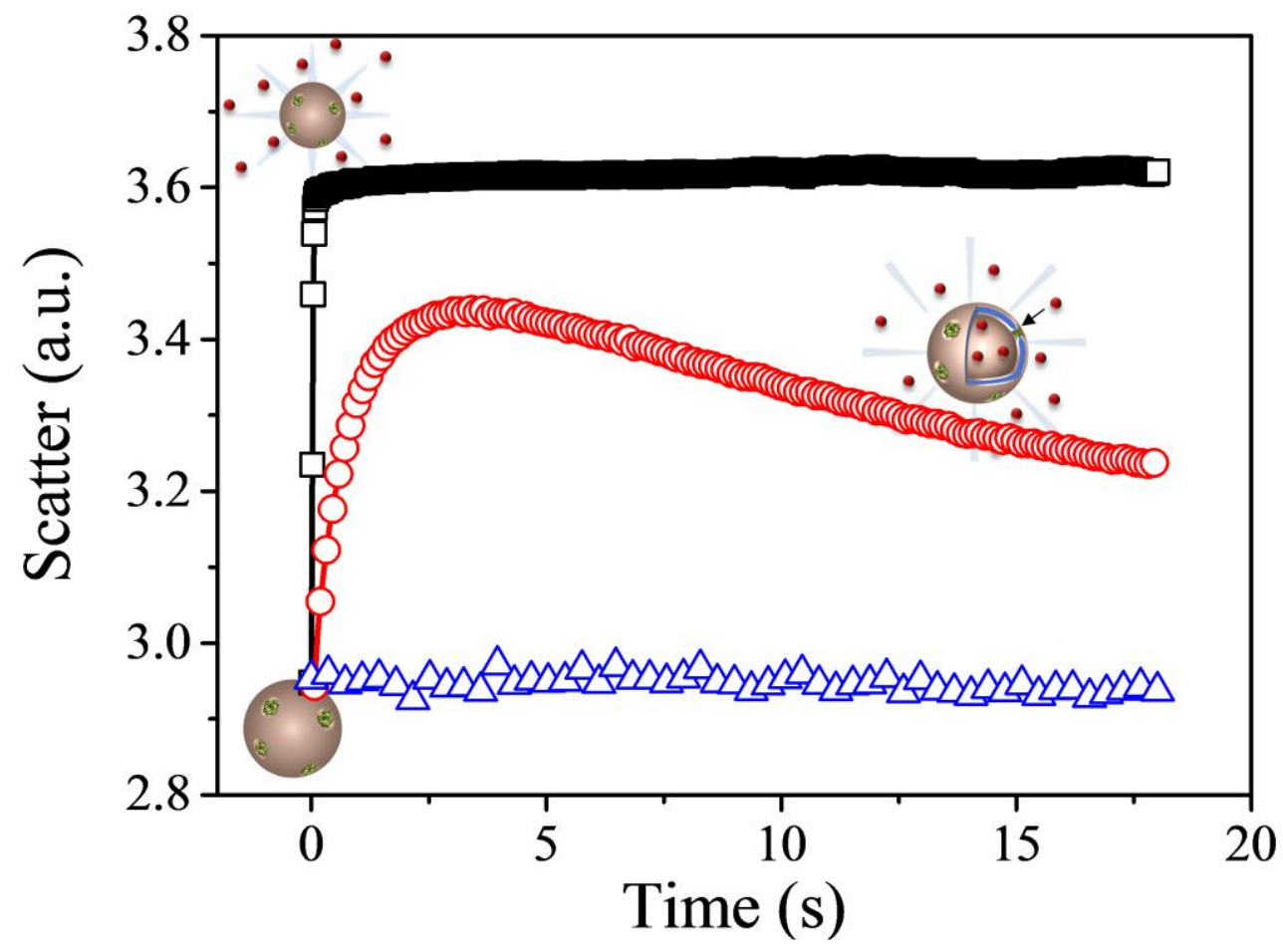


A

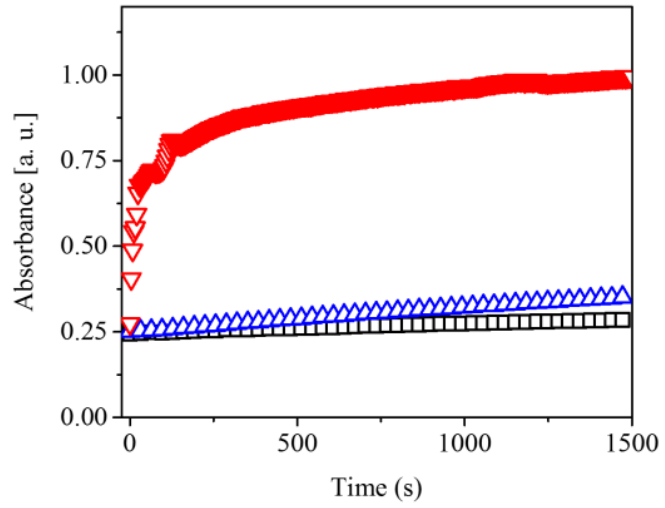

B

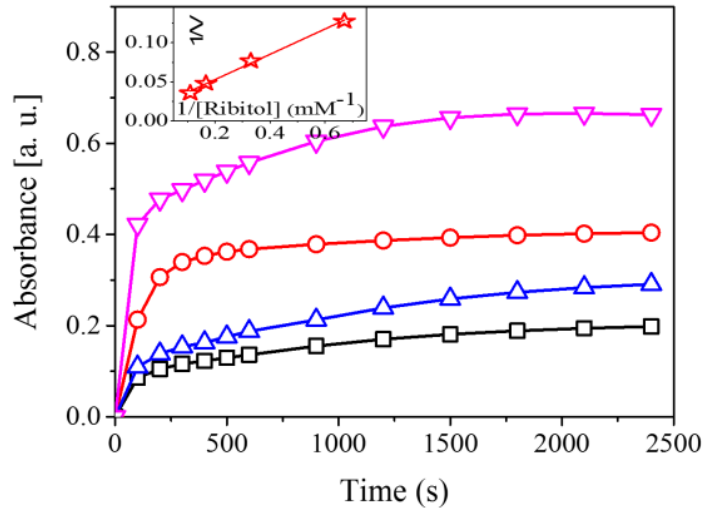


A

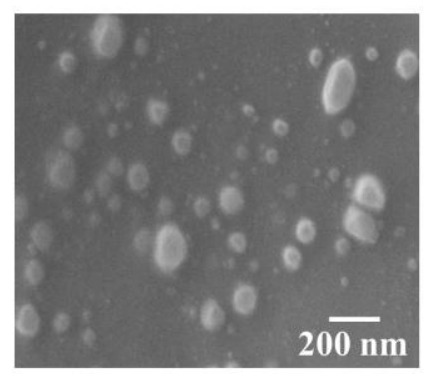

B

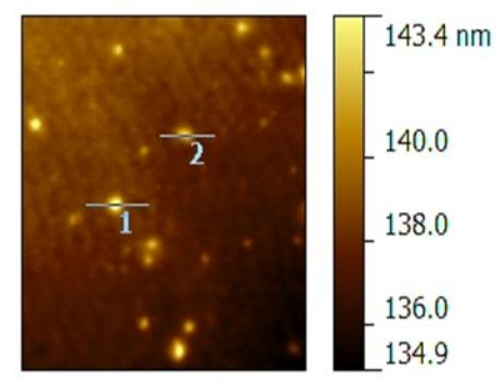

C

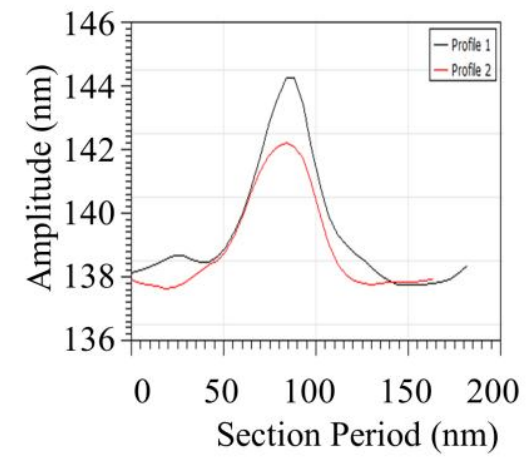




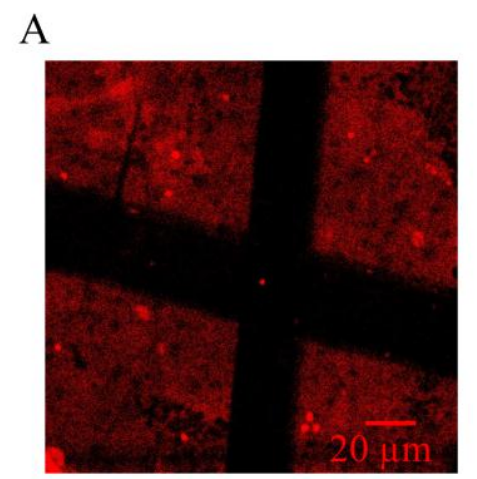

B

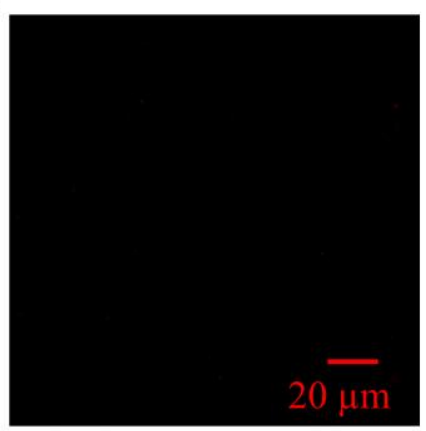

C

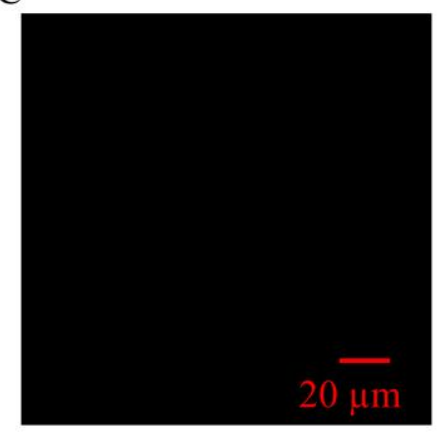




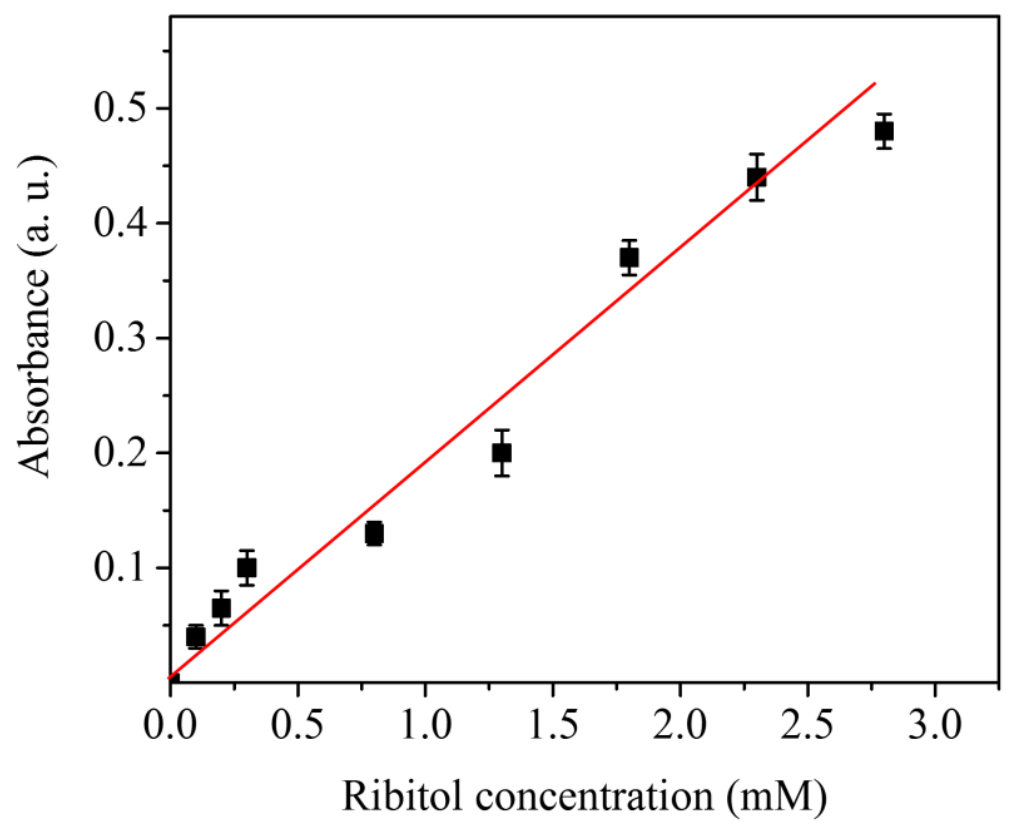

